\title{
UNA CONTRIBUCIÓN AL CORPUS FILOSÓFICO COLONIAL CHILENO: LOS CURSOS DE ANIMÁSTICA*
}

\section{A CONTRIBUTION TO THE CORPUS OF COLONIAL CHILEAN PHILOSOPHY: THE ANIMASTICS COURSES}

\author{
ABEL ARAVENA ZAMORA**
}

RESUMEN: El siguiente texto pretende contribuir a la identificación y difusión de una parte del corpus filosófico de la época colonial chilena: los cursos dedicados al estudio de la animástica. Se abordan las fuentes primarias conservadas en el Archivo Nacional Histórico y el Archivo de la Provincia Franciscana (Santiago de Chile) y se comenta brevemente su estructura, contenidos y particularidades. Con ello, se ofrece material, hasta ahora inédito, que facilitará y orientará futuras investigaciones en esta aún poco explorada área de la disciplina filosófica.

Palabras Clave: Animástica, corpus animasticum, filosofía colonial, filosofía chilena, historia de la filosofía.

Abstract: This paper aims to contribute to the identification and diffusion of an element of the philosophical corpus that emerged from colonial Chile: the courses dedicated to the study of the animastics. The paper first addresses the primary sources preserved in the National Historical Archive and in the Franciscan Historical Archive in Santiago, Chile, and subsequently provides a brief commentary of the structure, contents and particularities of these documents. Thus, this paper offers an approach to unpublished material, which aims to facilitate and guide future research in this relatively unexplored area of the discipline.

Keywords: Animastics, Corpus animasticum, colonial philosophy, Chilean philosophy, history of philosophy.

Recibido: 06.04.18. Aceptado: 26.03.19.

* Este trabajo es parte del proyecto Fondecyt Postdoctorado 3170144 "Animástica en el Chile colonial: la enseñanza jesuítica en los cursos manuscritos de los siglos XVII y XVIII”. El autor agradece la valiosa colaboración de Pedro González Cancino, del Archivo Nacional Histórico de Santiago de Chile.

** Doctor en Filosofía Contemporánea y Estudios Clásicos por la Universidad de Barcelona, España. Investigador del Centro de Estudios Avanzados de la Universidad de Playa Ancha, Valparaíso, Chile. Correo electrónico: abel.aravena@upla.cl 


\section{INTRODUCCIÓN}

TNO DE LOS aspectos más desconocidos dentro de la tradición filosófica chilena es el quehacer de la disciplina durante la época colonial. Pues, en efecto, este es un ámbito de investigación que en gran medida se encuentra aún inexplorado ${ }^{1}$.

Lo anterior se debe, entre otras muchas razones, al modesto número de obras conservadas en la actualidad; a su estado de deterioro, en mayor o menor grado, debido al paso del tiempo y a las condiciones particulares de conservación; a la dificultad en la comprensión de sus contenidos, pues se requiere no solo de un conocimiento mínimo de los temas filosóficos abordados, sino también de nociones básicas de la lengua y paleografía latina. En este sentido, difícilmente podrían sacarse conclusiones definitivas respecto a su originalidad, apego a la tradición o profundidad en el análisis de las materias.

El Archivo Nacional Histórico de Santiago de Chile (ANH) resguarda una de las colecciones de obras coloniales más ricas e importantes de Latinoamérica; sin embargo, en lo que atañe a la filosofía, no han sido objeto de estudio sistemático y, lamentablemente, en su gran mayoría se encuentran aún inéditas ${ }^{2}$. Por ello, la finalidad de este trabajo es rescatar las fuentes primarias inéditas del patrimonio filosófico nacional, contribuir al esbozo inicial de una parte importante del corpus filosófico de la época y, por último, presentar material suficiente para el desarrollo de futuras investigaciones en el área.

\section{CORPUS ANIMASTICUM}

En líneas generales, la animástica puede definirse como una aproximación especulativa al estudio de aquellas materias relativas al alma. En este sentido, la disciplina se enfocaba en estudiar, por ejemplo, las diferentes potencias, propiedades y pasiones del alma desde un punto de vista estrictamente racional. Por ello, si bien no había entonces una clara división entre la psi-

\footnotetext{
${ }^{1}$ Al respecto véase las clásicas obras de Hanisch (1963); Quiles (1953); Medina (1905) y Fuenzalida (1903).

${ }^{2}$ De hecho, en comparación con el trabajo realizado sobre el quehacer filosófico colonial en los países del cono sur americano, es evidente que, en Chile, este es un campo aún por explorar (Cf. Aravena Zamora (2015, pp. 289-90); Hofmeister Pich (2015); Hofmeister Pich, Lázaro y Culleton (2012) y Redmond (1972)).
} 
cología y lo que ahora se denomina biología, puede considerársele como la precursora de la psicología experimental o científica ${ }^{3}$.

Lo anterior se ve reflejado con distintos matices y variable extensión en las obras animásticas de la época colonial chilena. Pues, por un lado, dan cuenta de las características formales de la enseñanza de la disciplina en aquellos tiempos, esto es, copias en un latín muy particular de los cursos al dictado del profesor, estructura en forma de comentarios, disputaciones, cuestiones y secciones de estudio sobre los textos canónicos, etc. Y, por otro lado, en un nivel de contenidos, dan cuenta de la presencia de diversas interpretaciones medievales a las doctrinas aristotélicas sobre el alma.

Este último punto resulta de altísimo interés, pues pone de manifiesto las diferentes disputaciones y controversias teóricas de los profesores locales con las autoridades doctrinarias de las principales órdenes religiosas en los grandes temas de la tradición filosófica medieval ${ }^{4}$. Además, entrega valiosa información con respecto a los autores y obras predominantes en la organización de los contenidos curriculares específicos de la cátedra de Artes.

Por otra parte, y desde una perspectiva amplia, hay que señalar que los cursos de animástica estudian con detalle diversos temas acerca de la naturaleza humana, fundamentalmente a partir de la interpretación suareciana que predominaba entonces en la Compañía de Jesús. Entre las temáticas con frecuencia desarrolladas hallamos, por ejemplo, la definición del alma, sus distintos tipos, potencias, facultades y pasiones; la relación entre el alma y el cuerpo; el rol de la materia prima y las formas substanciales; $y$ la formación del compuesto humano. También hallamos temáticas desarrolladas por autores renacentistas, vinculadas con problemas de la cognición, emoción y volición: el proceso del conocimiento sensible e inteligible; la naturaleza y función del entendimiento, de las especies inteligibles y la imaginación; la libertad humana y su relación con la voluntad; y también la inmortalidad y espiritualidad del alma racional.

1. Commentaria in tres libros De anima ad mentem nostri Subtilis Doctoris Duns Scoti Theologorum Principis [Comentarios a los tres libros Acerca del alma según la doctrina de nuestro Doctor Sutil Duns Escoto, Príncipe de los

\footnotetext{
${ }^{3}$ Cf. Aravena Zamora (2016a, pp. 480 ss); Sondag (2008, p. 32); Nussbaum y Oksenberg Rorty (2003) y Barnes, Schofield y Sorabji (1979).

${ }^{4}$ Cf. Aravena Zamora (2018).
} 
Teólogos]. Juan de Fuica, O. F. M., 1689. Archivo de la Provincia Franciscana.

Esta obra es el curso de animástica más antiguo del que tenemos conocimiento. Corresponde al último de los Comentarios Filosóficos ${ }^{5}$, que el franciscano chileno Juan de Fuica (ca 1659 - 17??) dictó en 1689 en el Colegio San Diego de Alcalá de Santiago de Chile, y comprende las últimas fojas del volumen (274r-297v). Probablemente, en su totalidad, el texto pretendía ser un manual de estudios de la Provincia Franciscana chilena.

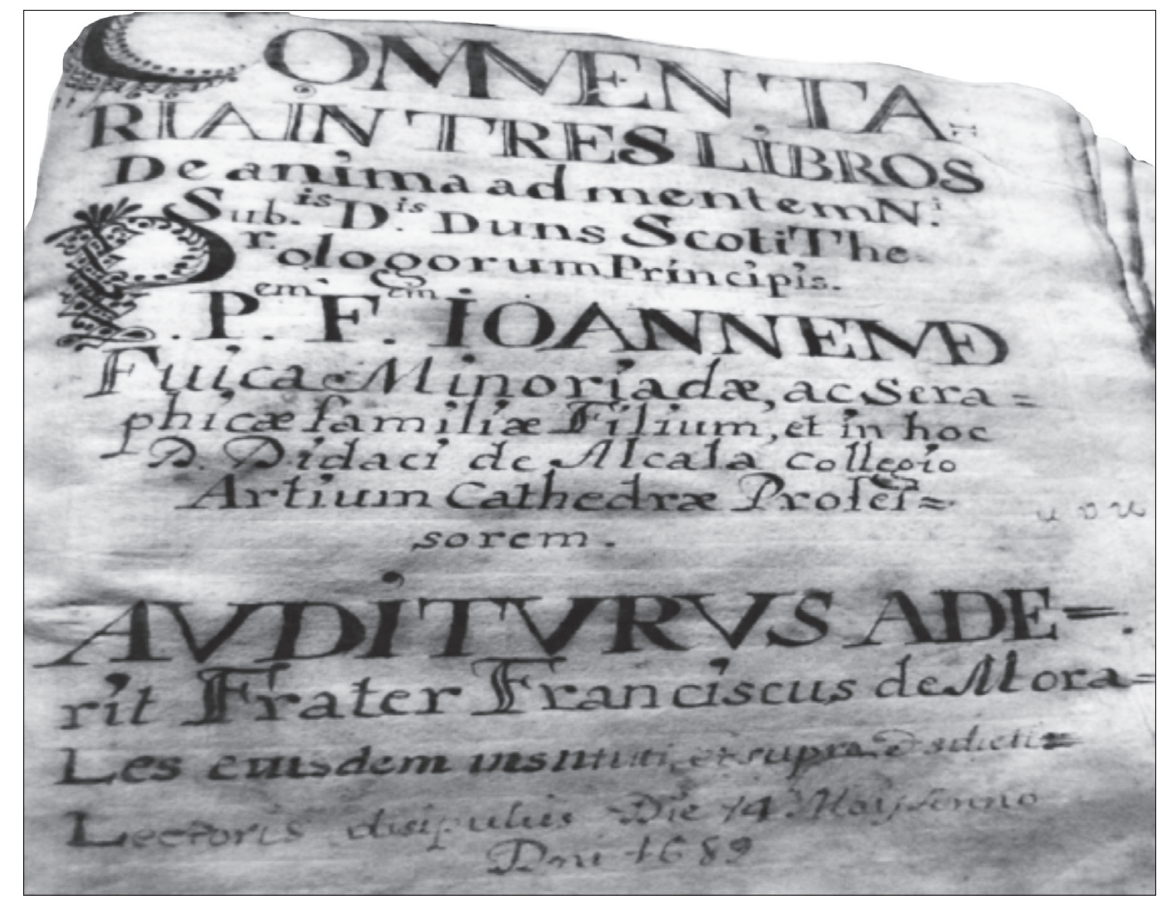

Figura 1. Portada de los Comentarios a los tres libros Acerca del alma de Juan de Fuica (f. 274r). Santiago, 1689. Archivo de la Provincia Franciscana.

Como hemos estudiado con mayor detalle en otros trabajos 6 , el volumen contiene las materias del curso trienal 1687-1689, dictado por el franciscano en dos instituciones que la orden mantenía en la ciudad de Santia-

\footnotetext{
${ }^{5}$ El título completo del volumen manuscrito es Commentaria phylosophica ad mentem Doctoris Subtilissimi Patris Fratris Joannis Duns Scoti Sacratissimi Ordinis Minorum et Theologorum Principis.

${ }^{6}$ Cf. Aravena Zamora (2016b, pp. 84-95); (2016a, pp. 478-485).
} 
go: el Convento Grande del Socorro y el Colegio San Diego de Alcalá. Por ello incluye también las Súmulas (3r-35r) y los comentarios a la Lógica (37r -94v), Metafísica (95r-162v), Física (163r-254v) y Acerca de la generación y la corrupción (256r-273v).

Específicamente, el curso de animástica ad mentem Scoti está estructurado en siete partes o distinciones, las que abordan distintos temas. En ellas, el fraile estudia detenidamente el alma en general (De anima in communi) y sus distintas potencias (De potentiis animae in communi); la esencia y potencias del alma vegetativa (De essentia animae vegetativa et illius potentiis); las potencias en general del alma sensitiva (De anima sensitiva et eius potentiis in communi); los sentidos externos e internos (De sensibus externis et internis in particulari); el alma racional (De anima rationali); y la potencia intelectiva (De potentia intellectiva).

Esta obra en particular constituye, indudablemente, un testimonio exclusivo y riquísimo de la tradición escotista durante la época colonial chilena. Por ello, remitimos al lector que desee mayor información sobre este texto a los artículos consignados en la respectiva nota al pie ${ }^{7}$.

2. Tractatus primus De anima, et eius potentiis [Tratado primero: Sobre el alma y sus potencias]. Manuel de Ovalle, S. J., 1707. Volumen 78, Fondo Antiguo, Archivo Nacional Histórico, ff. 248r-302r.

Manuel de Ovalle Ureta (1674-1736) desempeñó importantes cargos en las instituciones educativas de la Compañía de Jesús de Chile colonial. Durante tres años (1713-1715) fue Rector del Convictorio de San Francisco Javier y, luego, cumplió la misma labor en el Colegio de Concepción (1726). Producto de su temprana enseñanza en el Colegio Máximo San Miguel se conserva su Certamen de Lógica, Física y Metafísica (Logicae, Physicae et Metaphysicae Certamen $)^{8}$.

El tratado sobre el alma se halla en un volumen que no conserva su encuadernación original y en su portada se indica como perteneciente a la biblioteca del Colegio Máximo San Miguel. Si bien en el título de la obra no se menciona el tratado sobre el alma, este sí se encuentra señalado en un índice de contenidos al final del códice (f. 304v).

El curso se estructura en doce disputaciones. La primera (ff. 248r-251r) expone en tres secciones acerca de los conceptos de alma y vida (De anima,

${ }^{7}$ Cf. los trabajos de Hofmeister Pich, (2017; 2016); Guerrero (2014) e Iturriaga (1990).

${ }^{8}$ Cf. Hanisch (1963, p. 33). 
vitaque conceptibus); en la segunda (ff. 251r-254v) se estudia el proceso de información del alma (De anima informatione) y aborda, por ejemplo, si la sangre y las partes fluidas del cuerpo son informadas por el alma. La tercera (ff. $254 \mathrm{v}-257 \mathrm{r}$ ) se refiere a la igualdad y desigualdad de las almas ( $D e$ aequalitate et inaequalitate animarum); la cuarta (ff. 257r-260r), analiza las distintas potencias del alma en general (De potentiis animae in communi); en la quinta (ff. 260r-272r), se estudia en siete secciones la división del alma en vegetativa, sensitiva y racional (De divisione animae, multiplicique eius comparatio), comparando entre sí estos tres grados de los seres vivientes; mientras que en la sexta disputación (ff. 272r-273r) se estudian exclusiva y brevemente diferentes aspectos del alma racional (De anima rationalis). En este punto, Ovalle desarrolla temas relativos a su espiritualidad, indivisibilidad e inmortalidad.

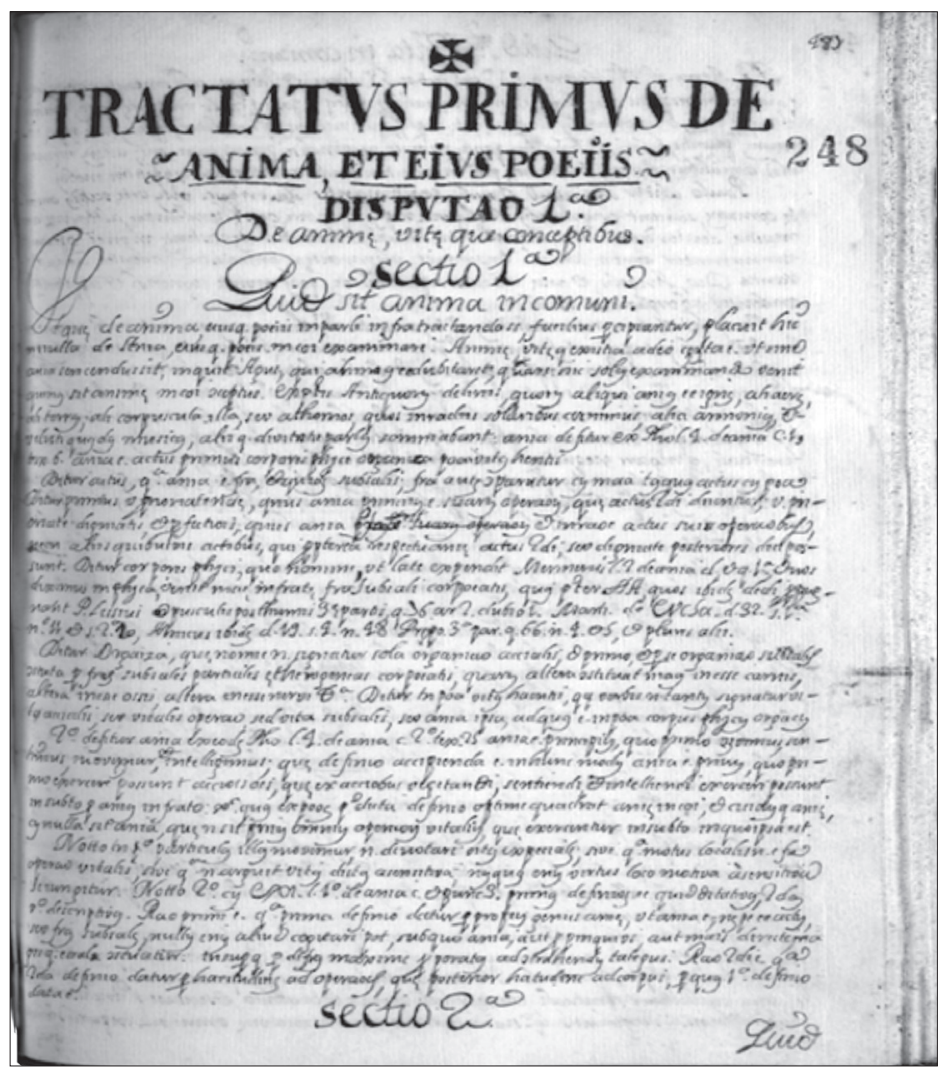

Figura 2. Portada del Tratado primero: Sobre el alma y sus potencias de Manuel de Ovalle (f. 248r). Santiago, 1707. Archivo Nacional Histórico. 
Posteriormente, en la disputación siguiente (ff. 273r-281v), el autor expone seis secciones sobre el intelecto y sus actos (De intellectu et eius actibus), abordando, entre otros temas, su dependencia de los fantasmas; si es posible una substancia intelectiva que no sea volitiva; en qué consiste la intelección; y sobre la aprehensión del verbo mental, el juicio y el discurso. Más adelante, en la octava disputación (ff. 281v-284v), analiza las propiedades de los actos del intelecto (De proprietatibus actuum intellectus) y estudia, por ejemplo, la oposición del asenso con el disenso, del amor con el odio y también de la opinión con la ciencia.

En la novena (ff. 284v-286v) estudia sobre la verdad y falsedad de los actos del intelecto (De veritate et falsitate actuum intellectus); en la décima (ff. 287r-292v) trata sobre la voluntad (De voluntate) y estudia el objeto, los actos de la voluntad y la omisión libre; mientras que en la undécima (ff. 292v-301v) expone sobre las especies y los hábitos (De speciebus et habitibus). Por último, en la disputación final del curso (ff. 301v-302r), en una sección única, Ovalle trata aspectos relativos al alma separada del cuerpo (De anima separata).

Nos parece muy importante señalar que, además de la total correspondencia de los contenidos estudiados, la fecha indicada en la foja 302r del volumen (Die 23 Augusti anni 1707) es exactamente la misma que la del curso anónimo que presentamos a continuación. Por ello, no cabe duda de que aquel es otra copia de este curso de Manuel de Ovalle, de modo que ya no es necesario considerarlo como una obra anónima.

3. Tractactus primus De anima eiusque potentiis [Tratado primero: Sobre el alma y sus potencias]. Anónimo S. J., 1707. Volumen 84, Fondo Antiguo, Archivo Nacional Histórico, ff. 76r-151v.

Este curso está incluido en el volumen titulado Philosophia Scholastica que contiene además, al inicio de la primera pieza, un tratado de Metafísica (ff. 1-75r). Luego, en una segunda pieza de extensión un poco menor, se incluye una obra escrita con otra mano y dedicada al estudio de la Lógica (ff. 152v-277r).

Como ya hemos indicado, tiene una correspondencia total de contenidos y fechas con la obra anterior. De acuerdo con esto, este curso es indudablemente una copia de las mismas clases dictadas en 1707 por el profesor jesuita Manuel de Ovalle en el Colegio Máximo San Miguel. No obstante, desconocemos hasta ahora los nombres de los alumnos copistas y/o propietarios de los volúmenes. Asimismo, habría que determinar los posibles 
efectos de la nueva encuadernación cartoné del volumen en el poco común ordenamiento de los contenidos de las dos piezas que incluye.

Por la razón ya señalada, indicamos aquí solo los nombres latinos y la extensión de cada una de las disputaciones del curso: De anima vitaeque conceptibus (ff. 76r-80r), De anima informacione (ff. 80v-84v), De aequalitate et inaequalitate animarum (ff. 85r-88v), De potentiis animae in communi (ff. 89r-92v), De divitione animae multiplicique eius comparatione (ff. 93r110r), De anima rationalis (ff. 110v-111v), De intellectu et eius actibus (ff. 112r-122v), De proprietatibus actuum intellectus (ff. 123r-127r), De veritate et falsitate actuum intellectus (ff. 127v-130v), De voluntate (ff. 131r-138r), De speciebus et habitibus (ff. 138v-150r), y De anima separata (ff. 150v-151v).

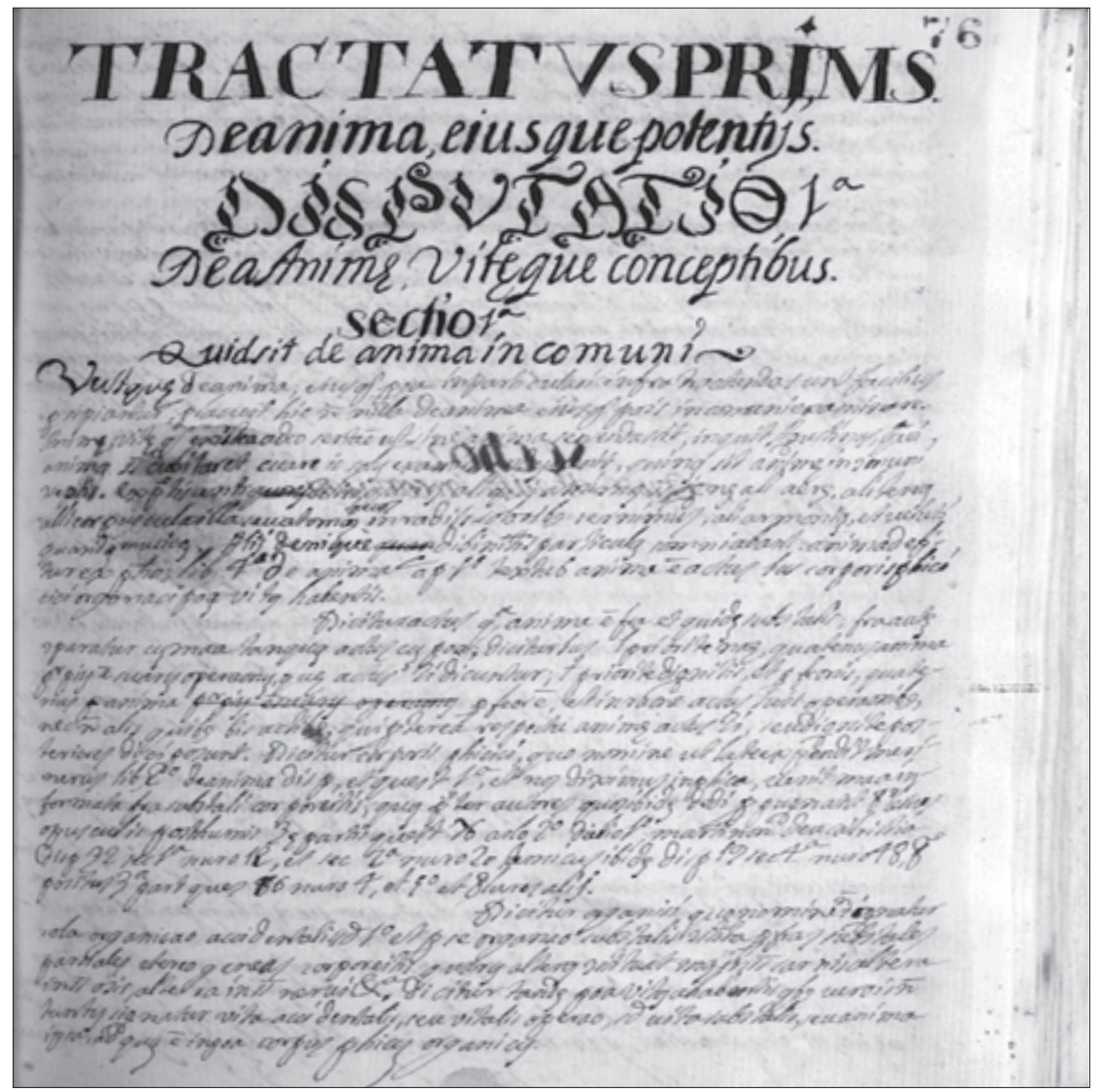

Figura 3. Portada del Tratado primero: Sobre el alma y sus potencias (f. 76r). Corresponde a una copia del curso de Manuel de Ovalle. Santiago, 1707. Archivo Nacional Histórico. 
4. Disputationes in Animasticam [Disputaciones de animástica]. Juan de Puga, S. J., 1710. Volumen 85, Fondo Antiguo, Archivo Nacional Histórico, ff. $2 \mathrm{r}-62 \mathrm{v}$.

Las disputaciones de animástica, que versan -según el mismo Puga (1682-1753) indica- sobre el cuerpo animado, corresponden a la primera parte del volumen. Pues, en efecto, el segundo tratado está dedicado a la Metafísica (Tractatus Metaphysicus, ff. 63r-118r), el tercero al estudio de los ocho libros de la Física (Disputationes in octo libros Physicorum Aristotelis, ff. 119r-281v) y, el cuarto y último, al tratado Acerca de la generación y de la corrupción (De generatione, sive in libros De orto et interitu, ff. $282 \mathrm{r}$ -291r). En las portadas del primero y el tercero, consta una dedicatoria a san Francisco Xavier, Apóstol de las Indias. Este volumen presenta también una encuadernación cartoné.

El curso de animástica fue dictado en el Colegio Máximo San Miguel ${ }^{9}$, en Santiago de Chile, y tuvo una duración de cuatro meses: desde el 20 de marzo al 28 de julio de 1710. Sus contenidos se organizan en siete disputaciones. La primera (ff. 2r-7r) expone brevemente diversos aspectos sobre el alma en general (De anima in communi) y explica los conceptos fundamentales de la disciplina (alma, cuerpo, espíritu y vida), recurriendo de manera concisa a las doctrinas de autores de la tradición griega como Tales, Demócrito, Pitágoras, Heráclito y Platón.

La segunda disputación (ff. $7 \mathrm{v}-8 \mathrm{v}$ ) estudia brevemente la información del alma en cuanto tal (De informatione animae ut sic); la tercera (ff. 9r-13v) se refiere a la igualdad de las almas (De aequalitate animarum) y estudia, por ejemplo, si todas las almas de la misma especie son iguales. En la cuarta disputación (ff. 14r-21v), el jesuita se enfoca en la multiplicidad de las almas (De multiplicitate animarum), profundizando en aspectos como los predicados quiditativos del alma, su indivisibilidad, espiritualidad e inmortalidad y también en algunas dificultades en torno al estado de separación. Más adelante, en la quinta disputación del tratado (ff. 22r-29v) aborda la distinción y operación de las potencias (De distinctione et operatione potentiarum), estudiando los sentidos externos en particular, el sentido interno, los sensibles y el apetito sensitivo.

La sexta disputación es la más extensa del curso (ff. 30r-59r). En ella Puga estudia acerca de las potencias espirituales (De potentiis spiritualibus) y dedica diferentes artículos para abordar en detalle numerosos temas. Entre ellos vale la pena mencionar: el intelecto y la intelección; si es posible

${ }^{9}$ Cf. Hanisch (1963, p. 33). 
que una substancia sea intelectiva y no volitiva; el verbo mental; la triple operación del intelecto; si puede darse en el intelecto el asenso y el disenso acerca de un mismo objeto; qué es la verdad y si es adecuadamente intrínseca con los actos; diferentes aspectos acerca de la ciencia, la fe y la opinión; y qué es la libertad.

Finalmente, en la séptima y última disputación (ff. 59v-62v), el profesor jesuita estudia las especies y los hábitos (De speciebus et habitibus). En este contexto, analiza, primero, qué son las especies, en cuáles potencias se dan y por qué razón concurren al acto del intelecto. Y, luego, expone acerca de las causas y la conservación de las especies. Por último, aborda qué son y en cuáles potencias se dan los hábitos y explica someramente en qué consiste la cualidad habitual.

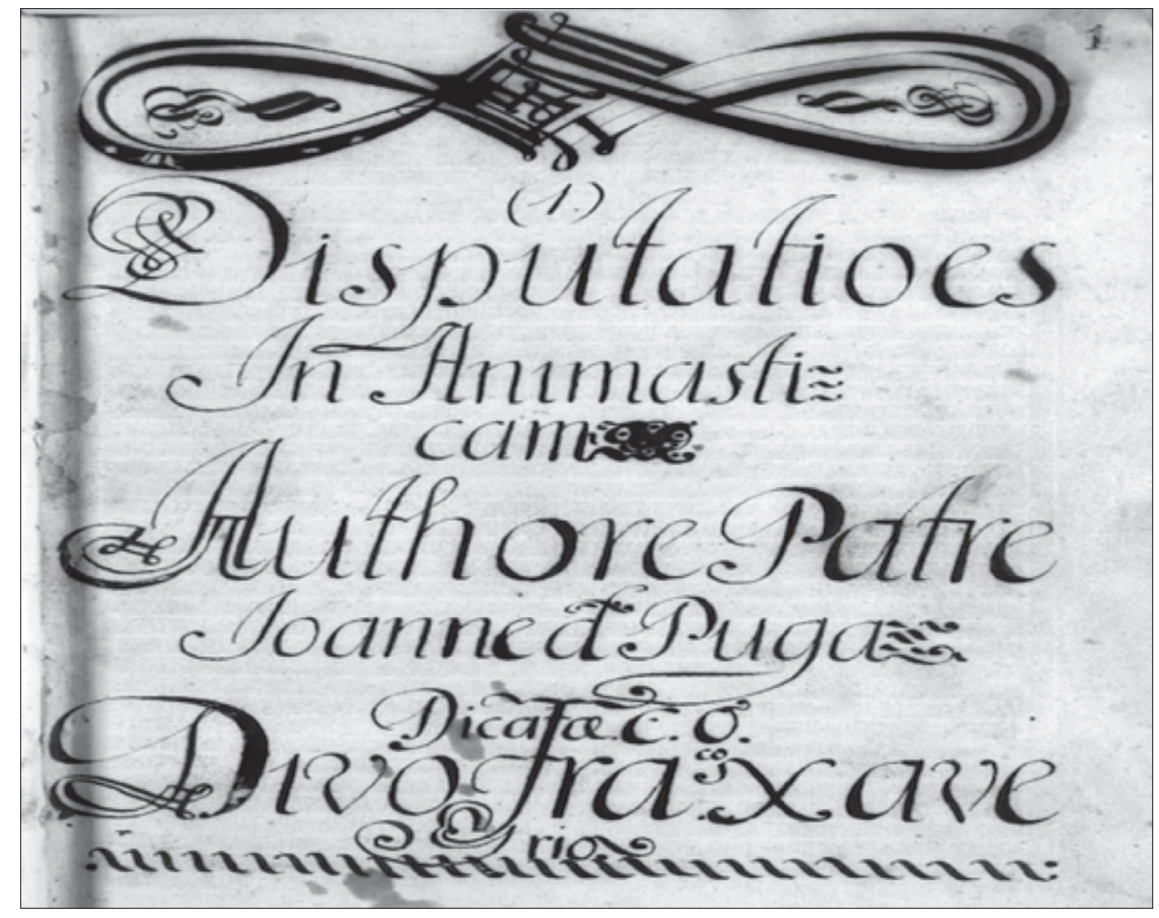

Figura 4. Portada de las Disputaciones de Animástica de Juan de Puga (f. 1r). Santiago, 1710. Archivo Nacional Histórico. 
5. Disputationes scholastica in tres libros Aristotelis De anima [Disputaciones escolásticas a los tres libros Acerca del alma de Aristóteles]. Anónimo S. J., 1715. Volumen 76, Fondo Antiguo, Archivo Nacional Histórico, ff. 101r-156r.

Este volumen incluye un total de tres obras: la primera estudia toda la metafísica del Estagirita (Disputationes in universam Aristotelis Metaphysicam, ff. 1r-99v); la segunda, la animástica, y la última se centra en el estudio del tratado Acerca de la generación y la corrupción (Disputationes in duos libros Aristotelis De ortu et interitu, ff. 157r-177r).

El curso de animástica es la segunda obra del volumen, que presenta encuadernación cartoné, y tiene una extensión total de 55 fojas numeradas, al igual que cada uno de sus parágrafos. Sus contenidos se estructuran en un proemio y tres libros. En el libro primero (ff. 101r-115r), el autor aborda la naturaleza del alma (De natura animae) en dos disputaciones. En la primera, estudia sobre la esencia, información y perfección del alma (De anima essentia eiusque informatione et perfectione) y define algunos conceptos fundamentales de la disciplina: cuerpo, espíritu y ser vivo. Se refiere, además, a la vida de las plantas, y a la igualdad y desigualdad de las almas, mientras que en la segunda disputación (De anima in specie), explica algunas propiedades características del alma racional (espiritualidad, indivisibilidad e inmortalidad), en virtud de su relación con el cuerpo y en estado de separación.

En el libro segundo (ff. 115r-126v), el autor estudia las potencias del alma, sus especies y hábitos (De potentiis animae. Speciebus et Habitibus) en tres disputaciones. En la primera explica las potencias del alma en general (De potentiis anima in communi) $\mathrm{y}$, especialmente, trata la polémica de si las potencias se distinguen realmente del alma. En la segunda aborda las potencias del alma en particular (De potentiis anima in particulari), refiriéndose aquí a los cinco sentidos externos, al sentido interno y al apetito sensitivo, y también al intelecto, la memoria y la voluntad. En la última disputación expone sobre la función de las especies impresas y los hábitos (De speciebus impressis et habitibus).

En el tercer y último libro, que es también el más extenso del curso (ff. 126r-156r), se estudian los actos vitales del alma (De actibus animae vitalibus) y los contenidos se desarrollan en tres disputaciones. La primera trata de la intelección, sus especies y pasiones (De intellectione, eiusque speciebus et affectionibus). Aquí, analiza en detalle en qué consiste formalmente la intelección y resuelve algunas objeciones, principalmente de tomistas como Cayetano, recurriendo a la autoridad de los conimbricenses y de jesuitas españoles como Arriaga, Alderete, Juan de Lugo y Ripalda. También revisa 
algunos aspectos acerca del verbo mental (De verbo mentis) y otras especies de intelección; y se pregunta si tanto el asenso y el disenso como la ciencia, la fe y la opinión pueden darse en el mismo intelecto a la vez en torno al mismo objeto.

En la segunda disputación, acerca de la verdad (De veritate), el autor profundiza en variados aspectos de este tema. Así, por ejemplo, estudia en qué consiste la verdad de conformidad (veritas comformitatis); si un acto una vez verdadero puede cambiar a falso; y también si las proposiciones de futuro contingente y condicionado son desde la eternidad una determinada verdad o falsedad. En la última disputación del libro estudia los distintos actos de la voluntad (De actibus voluntatis). En primer lugar, expone y divide el concepto de libertad; luego desarrolla si la libertad en acto segundo es extrínseca a los actos de la voluntad e indaga si es posible una pura omisión libre. Finalmente, el profesor de la Compañía resuelve objeciones de los autores tomistas sobre la voluntad. En apartados especiales, corrobora la propia conclusión de que una omisión pura no es un ejercicio de la libertad, y rechaza particularmente diez soluciones de los adversarios en torno a este polémico tema.

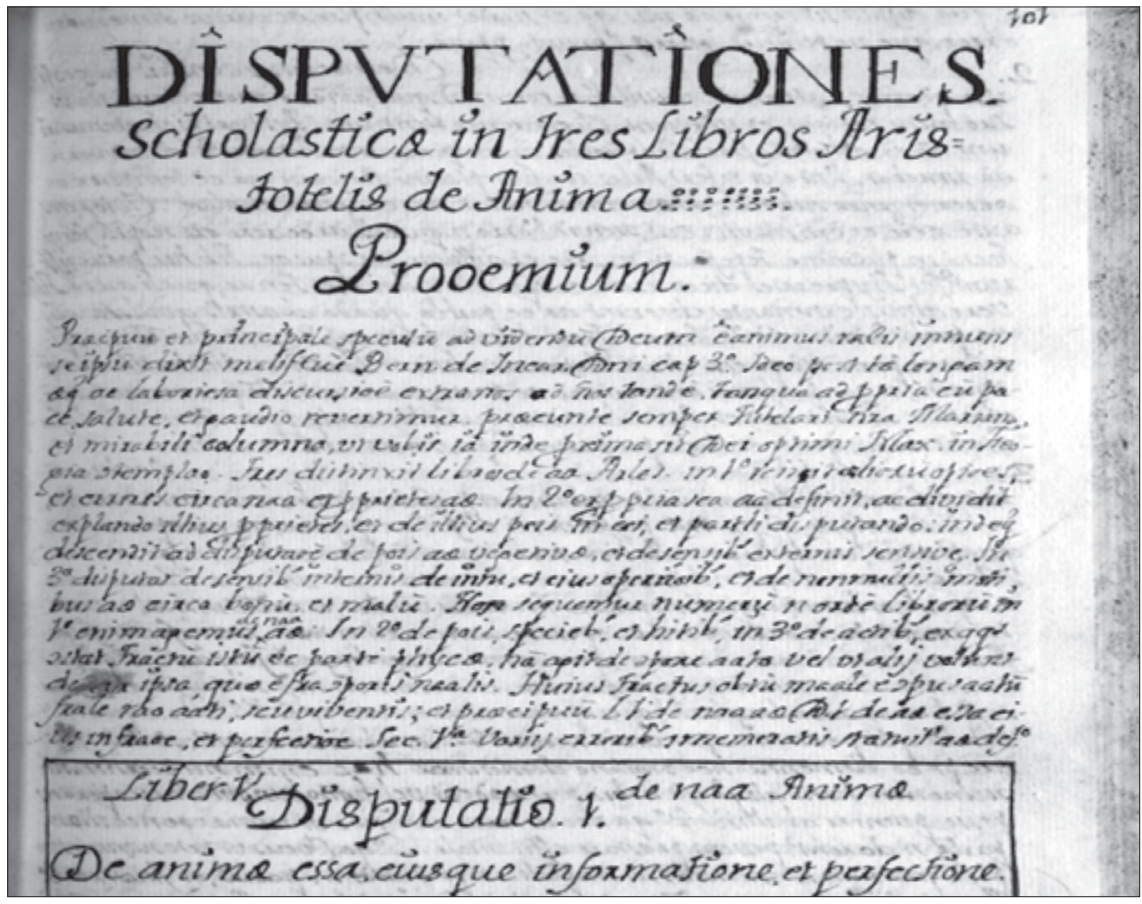

Figura 5. Portada de las Disputaciones escolásticas a los tres libros Acerca del alma de Aristóteles. Anónimo S. J. (f. 101r). Santiago, 1715. Archivo Nacional Histórico. 
6. Disputationes in libros De anima [Disputaciones a los libros Acerca del alma]. Miguel de Ureta, S. J., 1728. Volumen 91, Fondo Antiguo, Archivo Nacional Histórico, ff. 147r-209r.

El volumen 91 contiene dos obras: la primera, y más extensa parte del códice, está centrada en el estudio de la metafísica (Disputationes in Metaphysicam, ff. 2r-146v), y la segunda, en la animástica.

Esta última parte fue dictada durante el año 1728 por Miguel de Ureta S. J. (1694-1750), "publico philosophiae proffesore in alma Universitate Jacobopolitanae urbis Chilensis Regni”, en el Colegio Máximo San Miguel de la Compañía de Jesús ${ }^{10} \mathrm{y}$ fue copiado por el alumno José Ambert S. J., según él mismo lo indica en las portadas de las dos obras que contiene este volumen con encuadernación cartoné.

Los contenidos de animástica están estructurados en dos libros. El primero (ff. 147r-164v), acerca del alma en general y en particular (De anima in communi et in particulari), se desarrolla, a su vez, en dos disputaciones. En la primera de ellas estudia el concepto, información, perfección y divisibilidad del alma (De anima conceptu, informatione, perfectione et divisibilitate). Aquí, el profesor jesuita presenta, primero, las definiciones de alma y vida; la división del alma en corpórea y espiritual; y, luego, aborda las cuestiones de si almas de la misma especie pueden ser desiguales en cuanto a perfección entitativa. En este punto, para probar sus argumentos -frente a los Cayetano y Soto- recurre a destacados autores de la Compañía, como Vásquez, Molina, Toledo, Hurtado, Alfonso y Miguel de Viñas.

Más adelante, el jesuita chileno también aborda la cuestión de cuáles almas son realmente divisibles y en qué partes, valiéndose de la autoridad de Lince, Quiroz, Rubio, Aguilera, Oviedo, Ulloa, Compton y Viñas para discutir contra los argumentos de los autores tomistas. En la segunda disputación de esta parte, Ureta enseña además acerca de las potencias y los sentidos del alma y sus principios (De potentiis et sensibus animae, eorumque principiis). En este contexto, desarrolla la polémica cuestión de si las potencias se distinguen realmente del alma, fundamentando sus argumentos en autores de la Compañía como los ya mencionados para refutar la opinión de los seguidores del Preceptor Angélico. Por último, para finalizar el libro primero, Ureta se centra en el estudio de los cinco sentidos externos (vista, oído, olfato, gusto y tacto), de los sentidos internos del alma que se asientan en el cerebro (sentido común, facultad estimativa, fantasía, imaginación, memoria y reminiscencia) y del apetito sensible.

\footnotetext{
${ }^{10}$ Cf. Hanisch (1963, p. 33).
} 


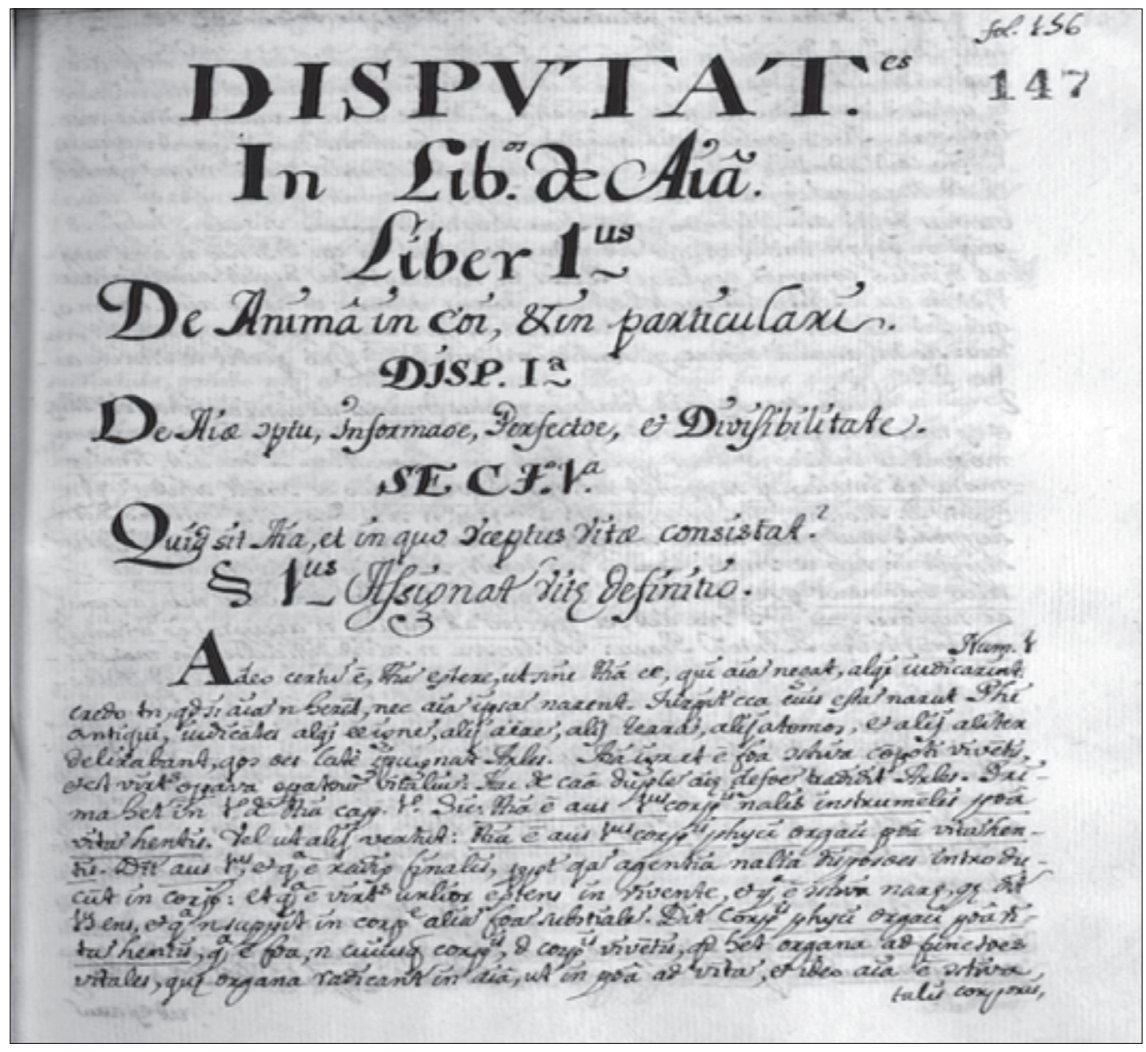

Figura 6. Portada de las Disputaciones a los libros Acerca del alma de Miguel de Ureta (f. 147r). Santiago, 1728. Archivo Nacional Histórico.

Por otro lado, el segundo libro del curso (ff. 165r-209r) versa sobre las perfecciones y actos del alma racional (De perfectionibus et actibus animae rationalis) y está organizado en cuatro disputaciones: la primera desarrolla algunas cuestiones acerca de las perfecciones del alma en estado de unión y separación del cuerpo; la segunda trata sobre la potencia y el acto del entendimiento, refiriéndose, entre otros temas, a las especies y hábitos intelectuales y a la naturaleza de la intelección. Aquí, nuevamente el profesor jesuita expone la opinión de los autores de la Compañía para refutar las objeciones de los autores tomistas. En la tercera disputación expone la división de los actos del intelecto, refiriéndose, entre otros temas, al verbo mental, a la aprehensión, al juicio y al discurso; mientras que en la cuarta y última disputación, Miguel de Ureta se refiere a los diferentes actos de la voluntad. 
7. De anima [Acerca del alma] ${ }^{11}$. Simón Fernández de Heredia, S. J., 1754. Volumen 92, Fondo Antiguo, Archivo Nacional Histórico, ff. 392v-426r.

Este curso se halla en la última parte del volumen titulado Philosophia Tripartita, que presenta encuadernación cartoné y ha sido atribuido al jesuita Simón Fernández de Heredia (1731-1769) ${ }^{12}$.

La primera obra está dedicada al estudio de la Lógica (ff. 1r-192v), la segunda a la Física (ff. 193r-316r), la tercera a la Metafísica (ff. 317r-392r) y la última parte, a pesar de no llevar un título indicativo, a la Animástica.

El inicio de este curso está incompleto, pues falta el comienzo de la primera disputación. En efecto, la primera indicación señala la cuarta sección $(397 \mathrm{v})$. La obra se estructura en tres disputaciones y un apéndice final. Por lo que hemos dicho, de la primera disputación (ff. 392v-402r) no conocemos el título ni los contenidos de las primeras tres secciones, pero en lo que resta de ella el autor estudia distintos aspectos del alma en general. Así, analiza si el alma racional es la verdadera forma espiritual del cuerpo, indivisible e inmortal; revisa distintos aspectos del alma separada del cuerpo; se refiere a la igualdad y desigualdad de las almas; $y$, por último, aborda la cuestión de si las partes del alma son o no heterogéneas.

La segunda disputación (ff. 402r-410r) se titula acerca de las potencias del alma y los hábitos (De potentiis animae et habitibus). En ella se refiere, entre otros temas, a la célebre polémica filosófica de si las potencias se distinguen realmente del alma, concluyendo -junto a los escotistas y muchos jesuitas, dice- que todas las potencias activas tomadas por sí mismas se identifican adecuada y realmente con el alma. En otra sección estudia las potencias materiales u orgánicas en particular, refiriéndose a las distintas funciones de la potencia vegetativa (nutrición, aumentación y generación), a la respiración y a la función del corazón en la circulación sanguínea; al sentido interno y a la fantasía o imaginación. Luego, estudia brevemente otros aspectos de las potencias materiales: al apetito material, el dolor, el llanto y la risa, los cinco sentidos externos, las especies intencionales y los hábitos.

La tercera y última disputación (ff. 410r-426r), trata de los actos de las potencias del alma (De actibus potentiarum animae). Aquí, el autor se refiere, primero, a las potencias espirituales del alma, vale decir, aquellas que son ejercidas por actos totalmente independientes del cuerpo como sujeto

\footnotetext{
${ }^{11}$ Si bien este título no aparece en el cuerpo del texto, los contenidos de animástica son desarrollados a partir de f. 392v.

${ }^{12}$ Cf. Hanisch (1963, pp. 63-64).
} 
de sustentación, y estudia diferentes aspectos del intelecto, la memoria y la voluntad.

Luego, analiza en qué consiste la intelección creada y se enfoca en la triple operacion de la mente, especialmente en las especies de intelección. Aquí, entre otras temáticas, el profesor jesuita se ocupa de si el juicio es indivisible; si en el mismo intelecto pueden darse a la vez el asenso y el disenso; si la ciencia, la fe y la opinión pueden conectarse en el mismo intelecto y en el mismo acto indivisible; si la verdad es adecuación dentro de los actos del intelecto y si un acto ya verdadero puede cambiar a falso; si y de qué modo la libertad en acto segundo es intrínseca a los actos de la voluntad; $y$, por último, si es posible una pura omisión libre.

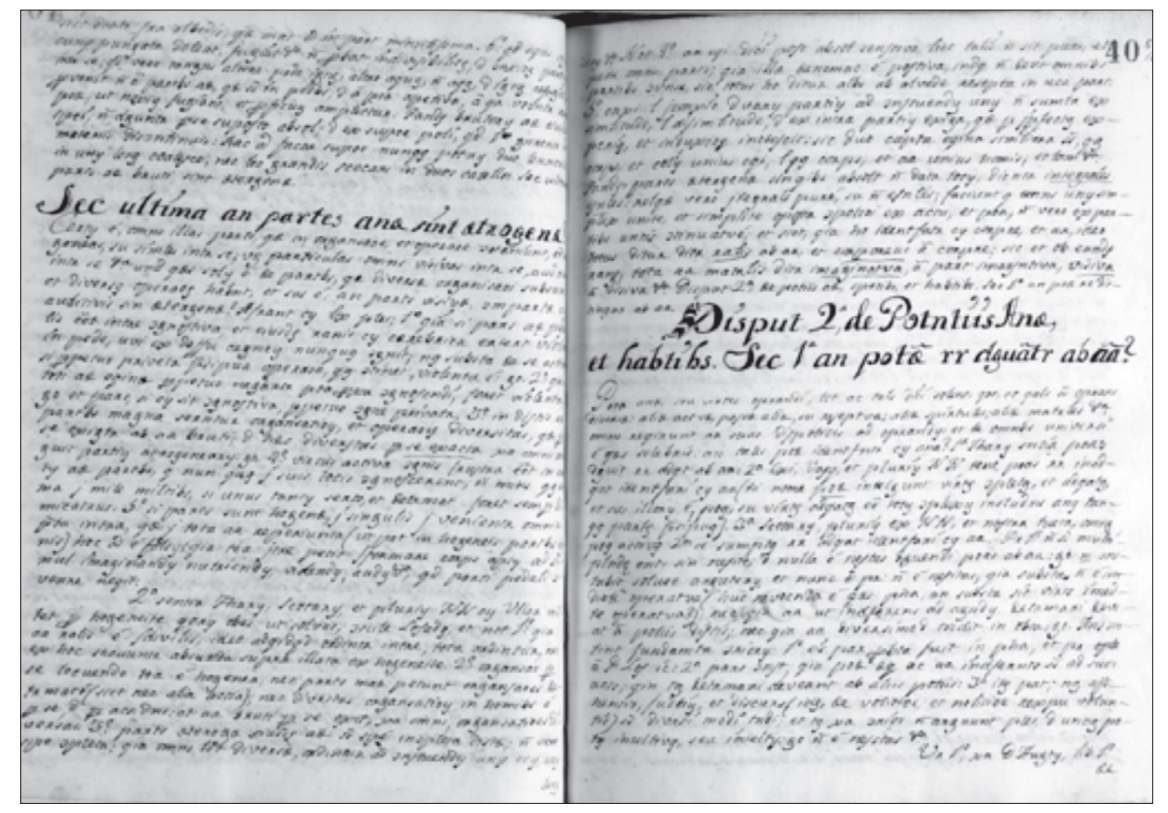

Figura 7. Detalle de algunos de los contenidos animásticos tratados en el curso (f. 402r). Santiago, 1754. Archivo Nacional Histórico.

\section{CONCLUSIONES}

Con este texto hemos intentado contribuir al conocimiento de un período aún en penumbra en cuanto al quehacer filosófico chileno se refiere. Para ello, hemos presentado las fuentes primarias de la enseñanza colonial de 
un área particular de la filosofía: la animástica, es decir, la disciplina que se centraba en el estudio especulativo del alma. Hemos identificado y descrito las obras conservadas en dos importantes fondos nacionales para esbozar preliminarmente esta importante parte del corpus filosófico de la época.

Hemos contribuido, además, a la identificación de una obra hasta ahora anónima como una copia del curso de 1707 de Manuel de Ovalle. Lo anterior permitirá, por ejemplo, contrastar aquellas obras anónimas que tratan sobre otras temáticas filosóficas (en especial las que abordan el estudio de la lógica, la física y la metafísica) con las del autor citado, pues probablemente existan algunas otras copias, aún sin identificar, de su enseñanza en el Colegio Máximo San Miguel de la Compañía.

Creemos también que el estudio detenido de las obras de animástica aquí ofrecidas permitirá el reconocimiento tanto de las fuentes filosóficas clásicas utilizadas como de las corrientes y autores más profundamente dominados por los profesores de aquella época en las aulas conventuales de estas latitudes.

En cuanto a los contenidos de los cursos presentados, hemos visto que en general se organizan en estructuras homogéneas y abordan determinados temas de manera recurrente. En este sentido, se halla como punto de partida de las reflexiones de los profesores jesuitas el legado aristotélico transmitido en sus definiciones del alma y las principales interpretaciones medievales y renacentistas de allí derivadas. Además, es manifiesto el foco de interés de los autores en el estudio del alma racional y sus diversas propiedades, en especial, el papel y la función del intelecto en el proceso de conocimiento humano.

Asimismo, estas obras de animástica registran y desarrollan las principales controversias filosóficas del período medieval europeo. Hallamos en sus páginas numerosas secciones dedicadas exclusivamente a discutir contra la argumentación de filósofos y teólogos tomistas, en especial en torno a la problemática de la esencia y la existencia, y la función del intelecto en el proceso del conocimiento. Este último punto es altamente importante, pues, además de ser hasta ahora un campo aún inexplorado, permitirá y guiará futuras líneas de investigación en esta área de la disciplina en particular.

De acuerdo con lo hasta aquí expuesto, creemos que el esfuerzo por sistematizar las obras filosóficas del período colonial en general y las de animástica en particular, poniendo así a disposición de los investigadores material hasta ahora inédito, constituye indudablemente un valioso aporte al conocimiento de la tradición filosófica nacional. 


\section{REFERENCIAS}

Aravena Zamora, A. (2015). Manuscritos filosóficos coloniales conservados en el Archivo Nacional Histórico de Santiago de Chile. Revista Española de Filosofía Medieval (22), 289-305.

Aravena Zamora, A. (2016a). Filosofía en el Chile colonial: el curso de animástica de fray Juan de Fuica. Anales del Seminario de Historia de la filosofía, 33(2), 475-488.

Aravena Zamora, A. (2016b). Un curso de filosofía del Chile colonial: los Comentarios Acerca del alma de fray Juan de Fuica (1689). Veritas (35), 81-98.

Aravena Zamora, A. (2018). La Física contra Tomistas de Lorenzo Romo S. J.: apuntes sobre una controversia filosófica en el Chile del siglo XVIII. Universum, 33(2), 53-73.

Barnes, J., Schofield, M., Sorabji, R. (Eds.). (1979). Articles on Aristotle. Volume 4: Psychology \& Aesthetics. London: Duckworth.

Fuenzalida, A. (1903). Historia del desarrollo intelectual en Chile (1541-1810). (Enseñanza Pública i Cultura Intelectual). Santiago de Chile: Imprenta Universitaria.

Guerrero, H. (2014). La escuela escotista en el Chile colonial. Alonso Briceño y Juan de Fuica. Revista Chilena de Estudios Medievales (6), 29-42.

Hanisch, W., S. J. (1963). En torno a la Filosofía en Chile (1594-1810). Santiago de Chile: Ediciones Historia, Universidad Católica de Chile.

Hofmeister Pich, R. (2015). Comission III: Latin Philosophy. Section 3: Scholastica colonialis. Bulletin de philosophie médiévale(56), 18-31.

Hofmeister Pich, R. (2016). A metafísica scotista de Ioannes de Fuica O.F.M. (séc. 17, Santiago de Chile): sobre o objeto da metafísica e a definiçấo de ente. Thaumàzein 9(18), 3-34.

Hofmeister Pich, R. (2017). Common real being and the scope of metaphysics according to fray Juan de Fuica O.F.M. (17th-18th centuries, Chile). Bulletin de philosophie médiévale (59), 247-284.

Hofmeister Pich, R., Lázaro, M., Culleton, A. (Eds.). (2012). Ideas sin fronteras en los límites de las ideas. Scholastica Colonialis: Status Quaestionis. Cáceres, España: Instituto Teológico "San Pedro de Alcántara" de Cáceres. Servicio de Publicaciones. Diócesis de Coria-Cáceres.

Iturriaga, R. (1990). El Escotismo en Chile. Anuario de Historia de la Iglesia en Chile, 8, 37-58.

Medina, J. T. (1905). La instrucción pública en Chile desde sus orígenes hasta la fundación de la Universidad de San Felipe. Santiago, Chile: Imprenta Elzeveriana.

Nussbaum, M. C., Oksenberg Rorty, A. (Eds.). (2003). Essays on Aristotle's De anima. Oxford: Oxford University Press.

Quiles, I. (1953). Manuscritos filosóficos en la época colonial en Chile. Ciencia y Fe, 39-61. 
Redmond, W. (1972). Bibliography of the Philosophy in the Iberian Colonies of America. The Hague, Netherlands: Martinus Nijhoff.

Sondag, G. (2008). Jean Duns Scot sur la connaissance intuitive intellectuelle (Cognitio Intuitiva). Veritas: Revista de Filosofía (Porto Alegre), 53, 32-58. 